\title{
Contents
}

\section{Gas Dynamics in Clusters of Galaxies}

C. L. Sarazin ..................................... 1

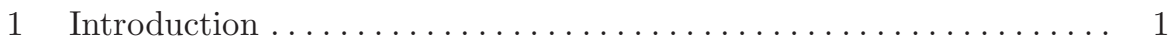

2 Physical State of the Intracluster Gas .................... 2

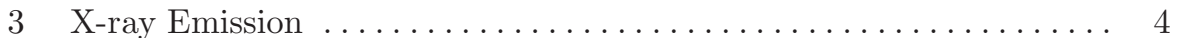

4 The Intracluster Medium as a Fluid $\ldots \ldots \ldots \ldots \ldots \ldots \ldots \ldots$

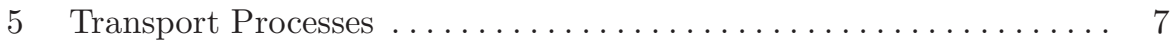

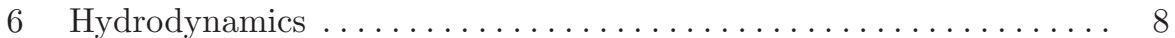

$7 \quad$ Hydrostatic Equilibrium ............................ 9

8 Cluster Masses ................................ 12

$9 \quad$ Heating and Cooling of Intracluster Gas .................... 14

10 Cluster Mergers ................................ 18

11 Thermal Physics of Merger Shocks..................... 18

12 Mergers and Cool Cluster Cores .......................... 23

References .................................. 29

Dynamics of the Hot Intracluster Medium

C. Jones, W. Forman, A. Vikhlinin, M. Markevitch, M. Machacek

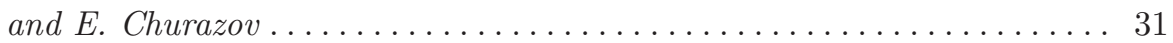

1 General Properties of Clusters . ....................... 31

2 Cooling and Feedback in Cluster Cores................... 37

3 Cluster Formation and Evolution .................... 52

4 The Effects of Clusters on Galaxies ..................... 56

5 Clusters and Cosmology ........................... 62

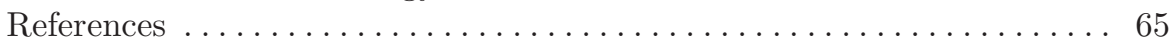

\section{Dynamics of Galaxies and Clusters of Galaxies}

L. A. Aguilar .................................. 71

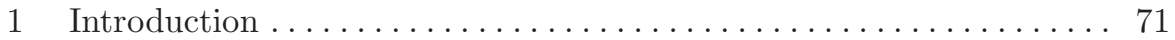

2 Basic Galactic Dynamics .......................... 72

3 A Case Study: The Navarro, Frenk and White profile .......... 75 


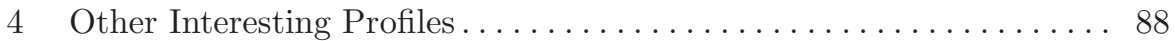

5 The Orbital Structure of Spherical Potentials . . . . . . . . . . . . . 90

6 A Sticky Story: Dynamical Friction . . . . . . . . . . . . . . . . . . . 95

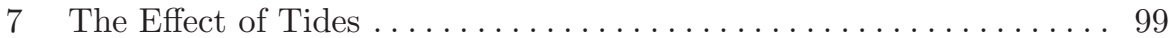

8 Tidal Encounters . . . . . . . . . . . . . . . . . . . . 103

9 Putting Things Together: The Orbital Decay of a Satellite within an Extended Halo . . . . . . . . . . . . . . . . . . . . . . . . . . . 108

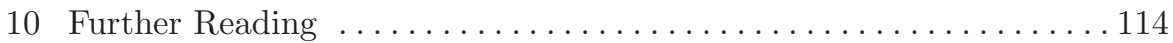

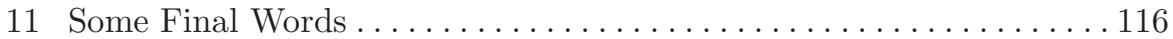

References ..................................... 117

\section{Optical Detection of Clusters of Galaxies}

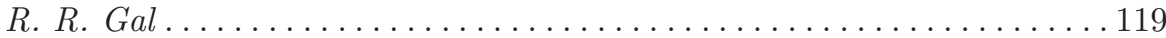

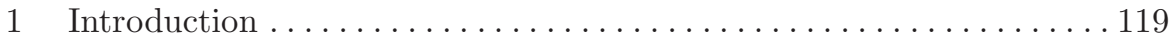

2 Photographic Cluster Catalogs . . . . . . . . . . . . . . . . . 120

3 Hybrid Photo-Digital Surveys . . . . . . . . . . . . . . . . . . . . . . . . . . . . . 122

4 Digital CCD Surveys . . . . . . . . . . . . . . . . . . . 123

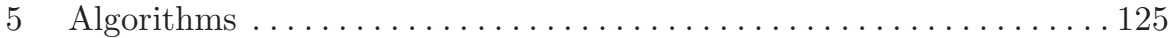

6 Conclusions..................................... 140

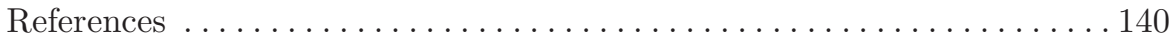

Clusters of Galaxies in the Radio: Relativistic Plasma and ICM/Radio Galaxy Interaction Processes

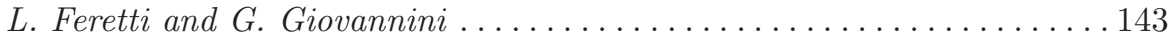

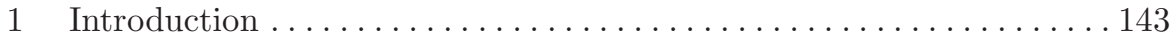

2 Basic Formulas from the Synchrotron Theory . . . . . . . . . . . . . . . . 144

3 Radio Emission from the ICM: Diffuse Radio Sources . . . . . . . . . . . . 149

4 Radio Emitting Particles . . . . . . . . . . . . . . . . . . . . . . . . . 156

5 Cluster Magnetic Fields . . . . . . . . . . . . . . . . . . . . . . . . 160

6 Radio Emission from Cluster Radio Galaxies . . . . . . . . . . . . . . . 167

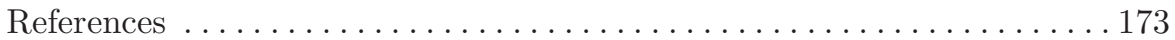

\section{Metal Content and Production in Clusters of Galaxies}

A. Renzini ...................................... 177

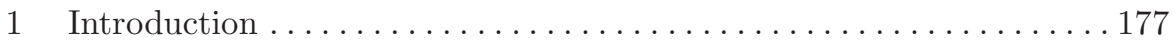

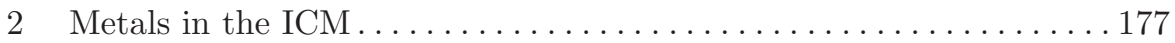

3 Metal Production: the Parent Stellar Population . . . . . . . . . . . . . . . 187

4 Metal Production: Type Ia vs. Type II Supernovae . . . . . . . . . . . . . . . 189

5 Metals from Galaxies to the ICM: Ejection vs. Extraction . . . . . . . . 198

6 Metals as Tracers of ICM Pre-heating . . . . . . . . . . . . . . . 200

7 Clusters vs. Field at $z=0$ and the Overall Metallicity of the Universe . . . . . . . . . . . . . . . . . . . . . . . . . . 201

8 Clusters vs. the Chemical Evolution of the Milky Way . . . . . . . . . . 204

9 Summary.................................. 206

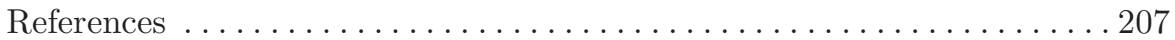




\section{Gravitational Lensing by Clusters of Galaxies}

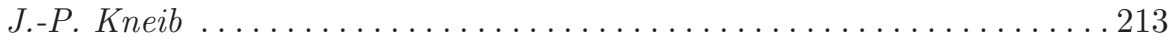

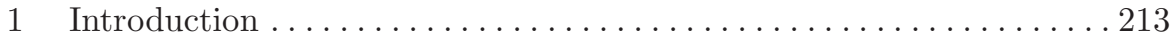

2 Historical Perspective . . . . . . . . . . . . . . . . . . . . . 213

3 Lensing Theory Useful in Cluster Lensing . . . . . . . . . . . . . . 217

4 Constraining the Cluster Mass Distribution . . . . . . . . . . . . . . . . 229

5 Clusters Lenses as Natural Telescopes . . . . . . . . . . . . . . . . . 245

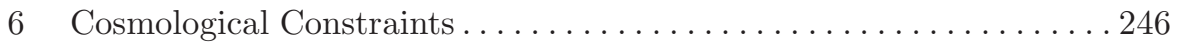

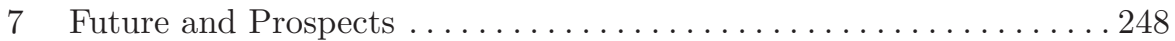

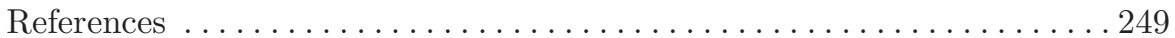

\section{The Sunyaev-Zel'dovich Effect in Cosmology} and Cluster Physics

M. Birkinshaw and K. Lancaster . . . . . . . . . . . . . . . . . . 255

1 Introduction . . . . . . . . . . . . . . . . . . . . . . . . 255

2 The Physics of the Sunyaev-Zel'dovich Effect . . . . . . . . . . . . . 256

3 Observing the Sunyaev-Zel'dovich Effect . . . . . . . . . . . . . . 263

4 Cluster Science from the Sunyaev-Zel'dovich Effect . . . . . . . . . . . . 273

5 Cosmology from the SZ Effect . . . . . . . . . . . . . . . . . . 277

6 The Next Generation of Instruments . . . . . . . . . . . . . . . . . . 281

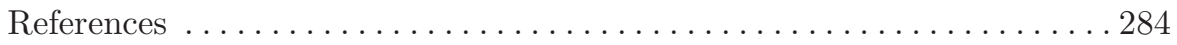

\section{Cosmology with Clusters of Galaxies}

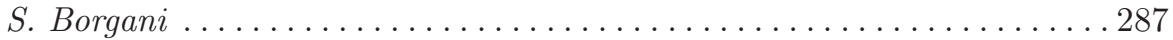

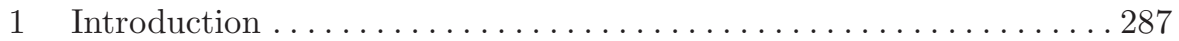

2 A Concise Handbook of Cosmic Structure Formation . . . . . . . . . . 289

3 The Mass Function . . . . . . . . . . . . . . . . . . . . . . 297

4 Building a Cluster Sample . . . . . . . . . . . . . . . . . . . . . . . 301

5 Methods to Estimate Cluster Masses . . . . . . . . . . . . . . . . . . . . . . 307

6 Constraints on Cosmological Parameters . . . . . . . . . . . . . . . 315

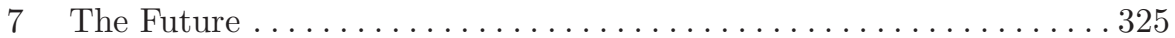

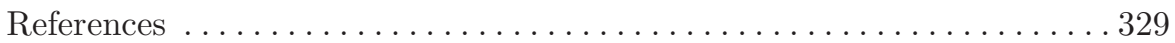

\section{Clusters and the Theory of the Cosmic Web}

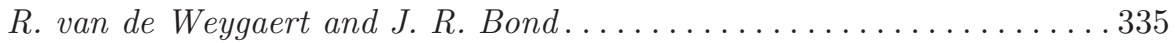

1 Outline: The Cosmic Web . . . . . . . . . . . . . . . . . . . 335

2 Cosmic Structure Formation: From Primordial Quantum Noise to the Cosmic Web. . . . . . . . . . . . . . . . . . . . . . . . 337

3 Hierarchical Structure Formation . . . . . . . . . . . . . . . . . . . . . . . . 349

4 Anisotropic and Weblike Patterns . . . . . . . . . . . . . . . . . 366

5 Conclusion: Clusters and the Cosmic Web . . . . . . . . . . . . . . . . . 404

6 Acknowledgments . . . . . . . . . . . . . . . . . . . . . . . . . 404

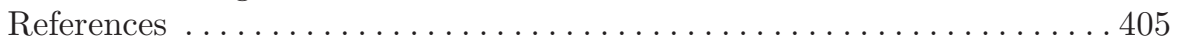


X Contents

\section{Observations and Morphology of the Cosmic Web}

Rien van de Weygaert and J. R. Bond....................409

1 Introduction . . . . . . . . . . . . . . . . . . . . . . . . . . . 409

2 The Emergence of the Cosmic Web .................... 411

3 Spatial Structure, Pattern Analysis and Object Identification . . . . . 432

4 Voids . . . . . . . . . . . . . . . . . . . . . . . . . . . . . . . . . . 442

5 Conclusion: Morphology of the Cosmic Web . . . . . . . . . . . . . . 460

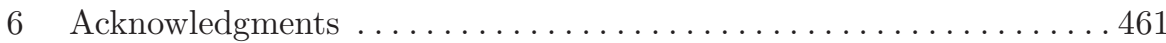

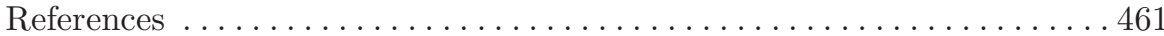

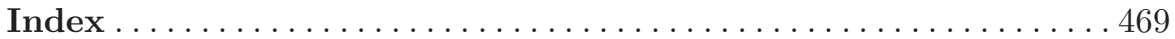

